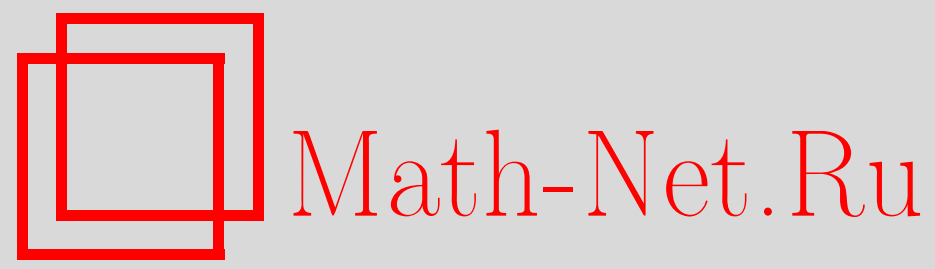

А. И. Аптекарев, В. Г. Лысов, Асимптотика $\gamma$-форм, генерируемых совместно ортогональными многочленами, Совр. пробл. матем., 2007, выпуск 9, 5562

DOI: https://doi.org/10.4213/spm17

Использование Общероссийского математического портала MathNet.Ru подразумевает, что вы прочитали и согласны с пользовательским соглашением

http://www . mathnet.ru/rus/agreement

Параметры загрузки:

IP : 54.224 .187 .69

26 апреля 2023 г., 05:15:22

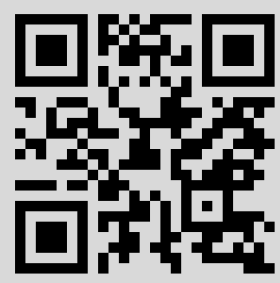




\title{
Асимптотика $\gamma$-форм, генерируемых совместно ортогональными многочленами ${ }^{1}$
}

\author{
А. И. Аптекарев, В. Г. Лысов
}

\section{1. Введение}

Рассмотрим постоянную Эйлера $\gamma$

$$
\gamma:=\lim _{n \rightarrow \infty}\left(\sum_{k=1}^{n} \frac{1}{k}-\ln (n)\right)=-\int_{0}^{\infty} \ln x e^{-x} d x .
$$

Интегрирование полиномиальных последовательностей $\left\{Q_{n}\right\}$ генерирует $\gamma$-формы

$$
f_{n}=p_{n}-\gamma q_{n}=\int_{0}^{\infty} Q_{n}(x) \ln x e^{-x} d x
$$

причем коэффициент при $\gamma$ в форме (1) равен

$$
q_{n}=\int_{0}^{\infty} Q_{n}(x) e^{-x} d x
$$

Настоящая работа посвящена получению асимптотики $\gamma$-форм генерируемых последовательностью многочленов

$$
Q_{n}(x)=\frac{1}{(n !)^{2}} \frac{e^{x}}{x-1}\left(\frac{d}{d x}\right)^{n} x^{n}\left(\frac{d}{d x}\right)^{n}(x-1)^{2 n+1} x^{n} e^{-x}
$$

Эти многочлены, впервые, появились в работе [1]. Они являются общими знаменателями рациональных аппроксимаций ЭрмитаПаде для системы из четырех функций

$$
\hat{\mu}_{k}(x):=\int_{0}^{1} \frac{w_{k}(x)}{z-x} d x, \quad \widehat{S}_{k}(x):=\int_{1}^{\infty} \frac{w_{k}(x)}{z-x} d x, \quad k=1,2,
$$

\footnotetext{
${ }^{1}$ Работа частично поддержана грантом научных школ НШ-1551.2003.1, программой № 1 ОМН РАН, грантами РФФИ-05-01-00522 и ИНТАС-03-516637.
}

(C) Аптекарев А. И., Лысов В. Г., 2007 
задаваемых весами

$$
w_{1}(x)=(1-x) e^{-x}, \quad w_{2}(x)=(1-x) \ln (x) e^{-x} .
$$

Результатом работы является

ТЕОРемА. Пусть $q_{n} u f_{n}$ определены подстановкой (3) в (2) и в (1) соответственно. Тогда справедливы асимптотические формульь

$$
\begin{aligned}
q_{n} & =(2 n) ! \frac{e^{\sqrt{2 n}}}{\sqrt[4]{n}}\left(\frac{1}{\sqrt{\pi}(4 e)^{3 / 8}}+\mathcal{O}\left(n^{-1 / 2}\right)\right), \\
f_{n}=p_{n}-\gamma q_{n} & =(2 n) ! \frac{e^{-\sqrt{2 n}}}{\sqrt[4]{n}}\left(\frac{2 \sqrt{\pi}}{(4 e)^{3 / 8}}+\mathcal{O}\left(n^{-1 / 2}\right)\right) .
\end{aligned}
$$

\section{2. Доказательство теоремы}

Идея доказательства состоит в получении контурных интегральных представлений для $q_{n}$ и $f_{n}$, с последующим применением метода перевала. Напомним основное соотношение метода перевала

$$
\int \psi(t) e^{n \varphi(t)} d t=\sqrt{\frac{2 \pi}{-n \varphi^{\prime \prime}\left(t_{0}\right)}} \psi\left(t_{0}\right) e^{n \varphi\left(t_{0}\right)}\left(1+\mathcal{O}\left(n^{-1}\right)\right)
$$

где контур интегрирования в левой части (5) и точка $t_{0}$ в правой части выбирается так, чтобы

$$
\varphi^{\prime}\left(t_{0}\right)=0, \quad \varphi^{\prime \prime}\left(t_{0}\right)\left(t-t_{0}\right)^{2}<0 .
$$

1. Асимптотика $q_{n}$. Вначале получим интегральное представление для последовательности $q_{n}$. Подставим выражение $(3)$ в (2) и применим формулу интегрирования по частям $2 n$ раз. Заметив, что все внеинтегральные члены равны нулю, получим

$$
q_{n}=\frac{(-1)^{n}}{n !} \int_{0}^{\infty}(x-1)^{2 n+1} x^{n} e^{-x}\left(\frac{d}{d x}\right)^{n} \frac{x^{n}}{(x-1)^{n+1}} d x
$$

Производную в последнем интеграле запишем в виде контурного интеграла по формуле Коши, имеем

$$
q_{n}=\frac{(-1)^{n}}{2 \pi i} \int_{0}^{\infty}(x-1)^{2 n+1} x^{n} e^{-x} d x \int_{\gamma_{x}} \frac{s^{n} d s}{(s-1)^{n+1}(s-x)^{n+1}},
$$




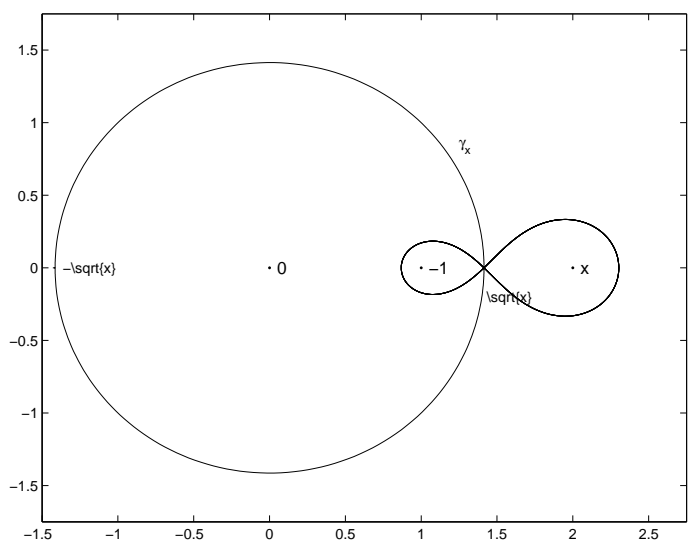

Рис. 6.1. Линии уровня и перевальный контур функции $\Phi_{1}$.

где $\gamma_{x}$ - контур, обходящий точку $x$ против часовой стрелки.

Найдем асимптотику внутреннего интеграла

$$
\int_{\gamma_{x}} \frac{s^{n} d s}{(s-1)^{n+1}(s-x)^{n+1}} .
$$

Обозначим,

$$
\Phi_{1}(s)=\frac{s}{(s-1)(s-x)}, \quad \psi_{1}(s)=\frac{1}{(s-1)(s-x)},
$$

при $x>0$. Функция $\Phi_{1}(s)$ имеет две седловые точки: $s= \pm \sqrt{x}$, в которых принимает значения: $\Phi_{1}( \pm \sqrt{x})=\frac{-1}{( \pm \sqrt{x}-1)^{2}}$. Контур $\gamma_{x}$ можно деформировать в линию уровня аргумента $\Phi_{1}(s)$ - окружность $|s|=\sqrt{x}$. На этой окружности функция $\Phi_{1}(s)$ принимает наибольшее по модулю значение в точке $\sqrt{x}$, а наименьшее в точке $-\sqrt{x}$. Значение второй производной $\ln \Phi_{1}$ в точке $\sqrt{x}$ равно

$$
\frac{\partial^{2} \ln \Phi_{1}}{\partial s^{2}}(\sqrt{x})=\frac{2}{\sqrt{x}(\sqrt{x}-1)^{2}} .
$$

По формуле (5)

$$
\int_{\gamma_{x}} \psi_{1}(s) \Phi_{1}^{n}(s) d s=\frac{(-1)^{n} \sqrt{\pi} i}{\sqrt{n}(\sqrt{x}-1)^{2 n+1} x^{1 / 4}}\left(1+\mathcal{O}\left(n^{-1}\right)\right) .
$$


Полученная асимптотическая формула справедлива равномерно по $x$ на компактных подмножествах из $\mathbb{R}_{+} \backslash\{1\}$. Однако, подставив ее в (6), убеждаемся, что точка перевала подынтегральной функции растет со скоростью порядка $n$. Чтобы проверить, что старший член асимптотики не изменится при сжатии $x=n y$, выпишем остаточный член. Сделаем замену переменной $e^{-z^{2}}=\left(\frac{\Phi_{1}(s)}{\Phi_{1}(\sqrt{x})}\right)^{n}$, тогда

$$
\begin{aligned}
\int_{\gamma_{x}} & \frac{s^{n} d s}{(s-1)^{n+1}(s-x)^{n+1}} \\
& =\frac{(-1)^{n} i}{\sqrt{n}(\sqrt{x}-1)^{2 n+1} x^{1 / 4}} \int_{-z_{*}}^{z_{*}} e^{-z^{2}} R^{-1 / 2}(z, x, n) d z,
\end{aligned}
$$

где функция $R$ имеет вид

$$
\begin{aligned}
& R(z, x, n)=\frac{e^{z^{2} / n}(\sqrt{x}-1)^{2}-2(x+1)+e^{-z^{2} / n}(\sqrt{x}+1)^{2}}{-4 \sqrt{x} e^{-z^{2} / n} z^{2} / n} \\
& =e^{z^{2} / n}\left(1+\sum_{k=1}^{\infty} \frac{z^{4 k}}{(2 k+1) ! n^{2 k}}-\frac{x+1}{2 \sqrt{x}} \sum_{k=0}^{\infty} \frac{z^{4 k+2}}{(2 k+2) ! n^{2 k+1}}\right),
\end{aligned}
$$

а $z_{*}>0$ определяется из уравнения

$$
e^{-z_{*}^{2}}=\left(\frac{\Phi_{1}(-\sqrt{x})}{\Phi_{1}(\sqrt{x})}\right)^{n}=\left(\frac{\sqrt{x}-1}{\sqrt{x}+1}\right)^{2 n} .
$$

Подставим (7) в выражение (6) для $q_{n}$ :

$$
\begin{aligned}
q_{n}= & \frac{(-1)^{n} n^{-1 / 2}}{2 \pi} \int_{0}^{\infty}(\sqrt{x}+1)^{2 n+1} x^{n-1 / 4} e^{-x} d x \\
& \times \int_{-z_{*}}^{z_{*}} e^{-z^{2}} R^{-1 / 2}(z, x, n) d z .
\end{aligned}
$$

Теперь сделаем замену $x=n y$ :

$$
\begin{aligned}
q_{n}= & \frac{n^{2 n+3 / 4}}{2 \pi} \int_{0}^{\infty}(\sqrt{y}+1 / \sqrt{n})^{2 n+1} y^{n-1 / 4} e^{-n y} d y \\
& \times \int_{-z_{*}}^{z_{*}} e^{-z^{2}} R^{-1 / 2}(z, n y, n) d z
\end{aligned}
$$


Из разложения (8) видно, что $R(z, n y, n)=1+\mathcal{O}\left(n^{-1 / 2}\right)$. Кроме того, из определения $(9) z_{*}$ следует, что $z_{*} \approx 2(n / y)^{1 / 4}$, поэтому

$$
\begin{aligned}
\int_{-z_{*}}^{z_{*}} & e^{-z^{2}} R^{-1 / 2}(z, n y, n) d z \\
& =\int_{-\infty}^{\infty} e^{-z^{2}} R^{-1 / 2}(z, n y, n) d z\left(1+\mathcal{O}\left(e^{-(n / y)^{1 / 2}}\right)\right) .
\end{aligned}
$$

Итак, имеем

$$
q_{n}=\frac{n^{2 n+3 / 4}}{2 \sqrt{\pi}} \int_{0}^{\infty}(\sqrt{y}+1 / \sqrt{n})^{2 n+1} y^{n-1 / 4} e^{-n y} d y\left(1+\mathcal{O}\left(n^{-1 / 2}\right)\right) .
$$

Асимптотику этого интеграла также найдем методом перевала. Пусть

$\varphi_{2}(y)=2 \ln (\sqrt{y}+1 / \sqrt{n})+\ln y-y, \quad \psi_{2}(y)=(\sqrt{y}+1 / \sqrt{n}) y^{-1 / 4}$.

Функция $\varphi_{2}$ достигает глобального максимума на полуоси в точке $y_{0}=2-1 / \sqrt{2 n}+3 /(8 n)+\mathcal{O}\left(n^{-3 / 2}\right)$. Имеем

$$
\begin{aligned}
& \varphi_{2}\left(y_{0}\right)=-2+2 \ln 2+\sqrt{\frac{2}{n}}-\frac{3}{8 n}+\mathcal{O}\left(n^{-3 / 2}\right), \\
& \varphi_{2}^{\prime \prime}\left(y_{0}\right)=-\frac{1}{2}+\mathcal{O}\left(n^{-1 / 2}\right), \\
& \psi_{2}\left(y_{0}\right)=2^{1 / 4}+\mathcal{O}\left(n^{-1 / 2}\right) .
\end{aligned}
$$

Таким образом, асимптотическая формула для $q_{n}$ имеет вид

$$
\begin{aligned}
q_{n} & =(2 n)^{2 n+1 / 4} e^{-2 n+\sqrt{2 n}-3 / 8}\left(1+\mathcal{O}\left(n^{-1 / 2}\right)\right) \\
& =\pi^{-1 / 2}(2 n) !(8 n)^{-1 / 4} e^{\sqrt{2 n}-3 / 8}\left(1+\mathcal{O}\left(n^{-1 / 2}\right)\right) .
\end{aligned}
$$

Первая формула в (4) получена.

2. Асимптотика $f_{n}$. Аналогично предыдущему пункту, получаем интегральное выражение для $f_{n}$ :

$$
\begin{aligned}
f_{n}= & \frac{1}{(2 \pi i)^{2}} \int_{0}^{\infty}(x-1)^{2 n+1} x^{n} e^{-x} d x \\
& \times \int_{\gamma_{x}} \frac{s^{n} d s}{(s-x)^{n+1}} \int_{\gamma_{s}} \frac{\ln t d t}{(t-1)(t-s)^{n+1}} .
\end{aligned}
$$


Преобразуем интеграл по $\gamma_{s}$. Контур $\gamma_{s}$ может быть деформирован в два берега отрицательной полуоси. Имеем

$$
\begin{aligned}
\int_{\gamma_{s}} \frac{\ln t d t}{(t-1)(t-s)^{n+1}} & =2 \pi i \int_{-\infty}^{0} \frac{d t}{(t-1)(t-s)^{n+1}} \\
& =(-1)^{n} 2 \pi i \int_{0}^{\infty} \frac{d t}{(t+1)(t+s)^{n+1}} .
\end{aligned}
$$

По теореме Фубини, поменяем порядок интегрирования в (10):

$$
\begin{aligned}
f_{n}= & \frac{(-1)^{n}}{2 \pi i} \int_{0}^{\infty}(x-1)^{2 n+1} x^{n} e^{-x} d x \\
& \times \int_{0}^{\infty} \frac{d t}{t+1} \int_{\gamma_{x}} \frac{s^{n} d s}{(t+s)^{n+1}(s-x)^{n+1}} .
\end{aligned}
$$

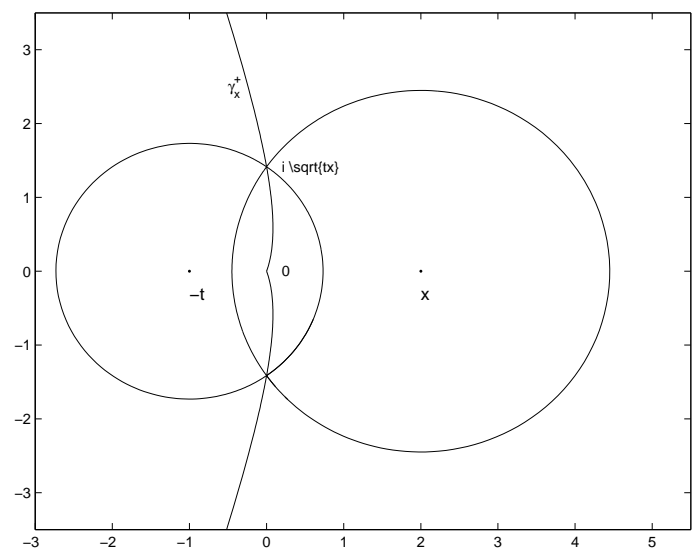

Рис. 6.2. Линии уровня и перевальный контур функции $\Phi_{3}$.

Найдем асимптотику внутреннего интеграла. Положим,

$$
\Phi_{3}(s)=\frac{s}{(s-x)(t+s)} .
$$

Функция $\Phi_{3}(s)$ имеет две точки перевала $s_{0}^{ \pm}= \pm i \sqrt{t x}$, в которых принимает значения равные по модулю. Деформируем контур $\gamma_{x}$ так, чтобы он проходил через перевальные точки в направлениях наискорейшего спуска. Тогда

$$
\int_{\gamma_{x}} \frac{s^{n} d s}{(t+s)^{n+1}(s-x)^{n+1}}=(I-\bar{I})
$$


где

$$
I=\int_{\gamma_{x}^{+}} \frac{s^{n} d s}{(t+s)^{n+1}(s-x)^{n+1}} .
$$

Сделаем замену переменной:

$$
e^{-z^{2}}=\left(\frac{\Phi_{3}(s)}{\Phi_{3}(i \sqrt{t x})}\right)^{n}
$$

После некоторых преобразований получим:

$$
I=\frac{(-1)^{n} n^{-1 / 2} e^{\pi i / 4}}{(1-i \sqrt{t / x})^{2 n+1} x^{n+3 / 4} t^{1 / 4}} \int_{-\infty}^{\infty} e^{-z^{2}} R^{-1 / 2}(z,-t / x, n) d z
$$

где

$$
\begin{aligned}
& R(z,-t / x, n)= \\
& \quad=\frac{e^{z^{2} / n}(1-i \sqrt{t / x})^{2}-2(1-t / x)+e^{-z^{2} / n}(1+i \sqrt{t / x})^{2}}{-4 i \sqrt{t / x} e^{-z^{2} / n} z^{2} / n} \\
& \quad=e^{z^{2} / n}\left(1+\sum_{k=1}^{\infty} \frac{z^{4 k}}{(2 k+1) ! n^{2 k}}-\frac{1-t / x}{2 i \sqrt{t / x}} \sum_{k=0}^{\infty} \frac{z^{4 k+2}}{(2 k+2) ! n^{2 k+1}}\right) .
\end{aligned}
$$

Обозначим,

$$
g(t)=\frac{e^{\pi i / 4}}{(1-i \sqrt{t / x})^{2 n+1} t^{1 / 4}} R^{-1 / 2}(z,-t / x, n)
$$

Будем считать, что ветви радикалов, входящих в определение $g(t)$, голоморфны в $\mathbb{C} \backslash \mathbb{R}_{+}$и положительны при подходе $\mathrm{k} \mathbb{R}_{+}$ сверху. Таким образом, $g(t)=g_{+}(t)$ при $t>0$. Легко видеть, что при таком определении ветвей $\bar{g}(t)=g_{-}(t)$ при $t>0$. Функция $g(t)$ аналитична в $\mathbb{C} \backslash \mathbb{R}_{+}$. Кроме того, $g(t) \rightarrow 0$ при $|t| \rightarrow \infty$. Поэтому для проведения в (11) интегрирования по $t$, имеем

$$
\begin{gathered}
\int_{0}^{\infty} \frac{(g(t)-\bar{g}(t)) d t}{t+1}=\left(\int_{(0,-\infty)_{+}}-\int_{(0,-\infty)-}\right) \frac{g(t) d t}{t+1} \\
=2 \pi i \operatorname{Res}_{t=-1} g(t)=\frac{2 \pi i}{(1+1 / \sqrt{x})^{2 n+1}} R^{-1 / 2}(z, 1 / x, n) .
\end{gathered}
$$


Подставляем найденное (см. (12)), (13), (14)) в выражение (11) для $f_{n}$ :

$$
\begin{gathered}
f_{n}=\frac{n^{-1 / 2}}{2 \pi} \int_{0}^{\infty}(\sqrt{x}+1)^{2 n+1} x^{n-1 / 4} e^{-x} d x \\
\times \int_{-\infty}^{\infty} e^{-z^{2}} R^{-1 / 2}(z, 1 / x, n) d z .
\end{gathered}
$$

Теперь делаем замену $x=n y$ и замечаем, что

$$
\int_{-\infty}^{\infty} e^{-z^{2}} R^{-1 / 2}(z, 1 / n y, n) d z=\sqrt{\pi}+\mathcal{O}\left(n^{-1 / 2}\right) .
$$

Имеем в (15)

$$
f_{n}=n^{2 n+3 / 4} \sqrt{\pi} \int_{0}^{\infty}(\sqrt{y}-1 / \sqrt{n})^{2 n+1} y^{n-1 / 4} e^{-n y} d y\left(1+\mathcal{O}\left(n^{-1 / 2}\right)\right) .
$$

Асимптотику этого интеграла также найдем методом перевала. Пусть

$$
\varphi_{4}(y)=2 \ln (\sqrt{y}-1 / \sqrt{n})+\ln y-y, \quad \psi_{4}(y)=(\sqrt{y}+1 / \sqrt{n}) y^{-1 / 4}
$$

Функция $\varphi_{4}$ достигает глобального максимума на полуоси в точке $y_{0}=2+1 / \sqrt{2 n}+3 /(8 n)+\mathcal{O}\left(n^{-3 / 2}\right)$,

$$
\begin{aligned}
& \varphi_{4}\left(y_{0}\right)=-2+2 \ln 2-\sqrt{\frac{2}{n}}-\frac{3}{8 n}+\mathcal{O}\left(n^{-3 / 2}\right), \\
& \varphi_{4}^{\prime \prime}\left(y_{0}\right)=-\frac{1}{2}+\mathcal{O}\left(n^{-1 / 2}\right), \\
& \psi_{4}\left(y_{0}\right)=2^{1 / 4}+\mathcal{O}\left(n^{-1 / 2}\right) .
\end{aligned}
$$

Таким образом, асимптотическая формула для $f_{n}$ имеет вид

$$
\begin{aligned}
f_{n} & =2 \pi(2 n)^{2 n+1 / 4} e^{-2 n-\sqrt{2 n}-3 / 8}\left(1+\mathcal{O}\left(n^{-1 / 2}\right)\right) \\
& =2 \pi^{1 / 2}(2 n) !(8 n)^{-1 / 4} e^{-\sqrt{2 n}-3 / 8}\left(1+\mathcal{O}\left(n^{-1 / 2}\right)\right) .
\end{aligned}
$$

Теорема доказана.

\section{Список литературы}

[1] A. I. Aptekarev, A. Branquinho, W. Van Assche, "Multiple orthogonal polynomials for classical weights", Trans. Amer. Math. Soc., 355:10 (2003), 3887-3914 doi 10.1090/S0002-9947-03-03330-0, MR 1990569, Zbl 1033.33002. 